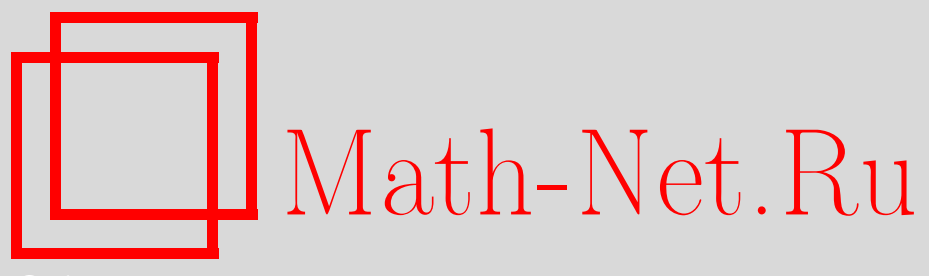

Р. Г. Новиков, Весовые преобразования Радона, для которых приближенная формула обращения Чанга является точной, УМH, 2011, том 66, выпуск 2, 237-238

DOI: https://doi.org/10.4213/rm9425

Использование Общероссийского математического портала Math-Net.Ru подразумевает, что вы прочитали и согласны с пользовательским соглашением http://www . mathnet.ru/rus/agreement

Параметры загрузки:

IP : 3.85 .5 .30

26 апреля 2023 г., 17:36:15

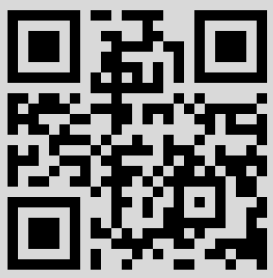




\section{Весовые преобразования Радона, для которых приближенная формула обращения Чанга является точной}

\section{Р. Г. Новиков}

Мы рассматриваем весовые лучевые преобразования $P_{W}$, определенные формулой

$$
P_{W} f(s, \theta)=\int_{\mathbb{R}} W\left(s \theta^{\perp}+t \theta, \theta\right) f\left(s \theta^{\perp}+t \theta\right) d t, \quad s \in \mathbb{R}, \quad \theta=\left(\theta_{1}, \theta_{2}\right) \in \mathbb{S}^{1},
$$

где $\theta^{\perp}=\left(-\theta_{2}, \theta_{1}\right), W=W(x, \theta)$ является весом, $f=f(x)$ - пробной функцией, $x \in \mathbb{R}^{2}$. $\mathrm{C}$ точностью до замены переменных, $P_{W}$ известно также как весовое преобразование Радона на плоскости.

Напомним, что в определении (1) произведение $\mathbb{R} \times \mathbb{S}^{1}$ интерпретируется, как множество всех ориентированных прямых в $\mathbb{R}^{2}$. Если $\gamma=(s, \theta) \in \mathbb{R} \times \mathbb{S}^{1}$, то $\gamma=\left\{x \in \mathbb{R}^{2}\right.$ : $\left.x=s \theta^{\perp}+t \theta, t \in \mathbb{R}\right\}$ (с точностью до ориентации) и $\theta$ дает ориентацию $\gamma$.

Мы предполагаем, что

$$
\begin{gathered}
W \text { комплекснозначна, } \quad W \in C\left(\mathbb{R}^{2} \times \mathbb{S}^{1}\right) \cap L^{\infty}\left(\mathbb{R}^{2} \times \mathbb{S}^{1}\right), \\
w_{0}(x) \stackrel{\text { def }}{=} \frac{1}{2 \pi} \int_{\mathbb{S}^{1}} W(x, \theta) d \theta \neq 0, \quad x \in \mathbb{R}^{2},
\end{gathered}
$$

где $d \theta$ - стандартный элемент длины на $\mathbb{S}^{1}$.

Если $W \equiv 1$, то $P_{W}$ известно как классическое лучевое преобразование (или преобразование Радона) на плоскости. Если

$$
W(x, \theta)=\exp (-D a(x, \theta)), \quad D a(x, \theta)=\int_{0}^{+\infty} a(x+t \theta) d t,
$$

где $a$ - некоторая достаточно регулярная комплекснозначная функция на $\mathbb{R}^{2}$, достаточно быстро зануляющаяся на бесконечности, то $P_{W}$ известно как лучевое преобразование (или преобразование Радона) с поглощением.

Классическое преобразование Радона возникает, в частности, в рентгеновской томографии. Преобразование Радона с поглощением (по крайней мере при $a \geqslant 0$ ) возникает, в частности, в однофотонной эмиссионной компьютерной томографии (со стандартной английской аббревиатурой SPECT). Некоторые другие веса $W$ также возникают в приложениях. Более подробную информацию по этим вопросам можно найти, например, в [1], [2].

Точные и одновременно явные формулы обращения для классического преобразования Радона и для преобразования Радона с поглощением были впервые даны в [3] и [4] соответственно. Для некоторых других весов $W$ точные и одновременно явные формулы обращения были даны в [5], [6].

С другой стороны, следующая приближенная формула обращения Чанга для преобразования $P_{W}$, где $W$ дано формулами (3) с $a \geqslant 0$, уже давно используется в численных расчетах, см. [7], [8], [2]:

$$
\begin{aligned}
& f_{\text {appr }}(x)=\frac{1}{4 \pi w_{0}(x)} \int_{\mathbb{S}^{1}} h^{\prime}\left(x \theta^{\perp}, \theta\right) d \theta, \quad h^{\prime}(s, \theta)=\frac{d}{d s} h(s, \theta), \\
& h(s, \theta)=\frac{1}{\pi} \text { p.v. } \int_{\mathbb{R}} \frac{P_{W} f(t, \theta)}{s-t} d t, \quad s \in \mathbb{R}, \quad \theta \in \mathbb{S}^{1}, \quad x \in \mathbb{R}^{2},
\end{aligned}
$$

где $w_{0}$ определено в (2). Известно, что формула (4) эффективна как первое приближение в SPECT-восстановлениях и, в частности, достаточно устойчива к сильному пуассоновскому шуму в SPECT-данных. 
Результаты настоящей заметки состоят в теореме 1 и замечании 1.

Пусть $C_{0}\left(\mathbb{R}^{2}\right)$ обозначает пространство непрерывных функций с компактным носителем на $\mathbb{R}^{2}$.

Теорема 1. Пусть предположения (2) выполнены и $f_{\text {appr }}(x)$ дано формулой (4). Тогда

$$
\left.f_{\text {appr }}=f \quad \text { (в смысле распределений }\right) \text { на } \mathbb{R}^{2} \text { для всех } f \in C_{0}\left(\mathbb{R}^{2}\right),
$$

если и только если

$$
W(x, \theta)-w_{0}(x) \equiv w_{0}(x)-W(x,-\theta), \quad x \in \mathbb{R}^{2}, \quad \theta \in \mathbb{S}^{1} .
$$

Схема доказательства теоремы 1 дана в [9] и использует, в частности, следующую формулу:

$$
\begin{gathered}
\frac{1}{2}\left(P_{W} f(s, \theta)+P_{W} f(-s,-\theta)\right)=P_{W_{\mathrm{sym}}} f(s, \theta), \quad(s, \theta) \in \mathbb{R} \times \mathbb{S}^{1}, \\
W_{\mathrm{sym}}(x, \theta)=\frac{1}{2}(W(x, \theta)+W(x,-\theta)), \quad x \in \mathbb{R}^{2}, \quad \theta \in \mathbb{S}^{1} .
\end{gathered}
$$

Утверждение, что при условиях теоремы 1 свойство (6) влечет тождество (5), может быть также выведено из рассмотрений, развитых в [2].

Используя то, что $W_{\text {sym }} \equiv w_{0}$ при условии (6), мы получаем также

ЗАмечание 1 . Пусть условия (2), (6) выполнены и $f \in C_{0}\left(\mathbb{R}^{2}\right)$. Тогда:

(A) $P_{W} f$ на $\Omega(D)$ однозначно определяет $f$ (или, более точно, $w_{0} f$ ) на $\mathbb{R}^{2} \backslash D$ с помощью (7) и восстановления Кормака по $P_{W_{\mathrm{sym}}} f$ на $\Omega(D)$, где $D$ - некоторый выпуклый компакт в $\mathbb{R}^{2}, \Omega(D)$ обозначает множество всех прямых в $\mathbb{R}^{2}$, которые не пересекают $D$;

(В) $P_{W} f$ на $\mathbb{R} \times\left(S_{+} \cup S_{-}\right)$однозначно определяет $f$ на $\mathbb{R}^{2}$ с помощью (7) и стандартного восстановления по данным с ограниченными направлениями $P_{W_{\text {sym }}} f$ на $\mathbb{R} \times S_{+}$, где $S_{+}$обозначает произвольное непустое открытое связное подмножество единичной окружности $\mathbb{S}^{1}, S_{-}=-S_{+}$.

В случае, когда $W$ дано формулами (3), при дополнительных условиях, что $a \geqslant 0$ и $\operatorname{supp} a \subset D$, где $D$ - некоторая известная не слишком большая область, и при $f \in C\left(\mathbb{R}^{2}\right), f \geqslant 0, \operatorname{supp} f \subset D$, преобразование $P_{W} f$ относительно хорошо приближается преобразованием $P_{W_{\text {appr }}} f$, где $W_{\text {appr }}(x, \theta)=w_{0}(x)+(W(x, \theta)-W(x,-\theta)) / 2$. При этом вес $W_{\text {appr }}$ уже удовлетворяет условию (6). Это объясняет эффективность формулы (4) как первого приближения в SPECT-восстановлениях (на уровне интегральной геометрии).

\section{Список литературы}

[1] F. Natterer, The mathematics of computerized tomography, Teubner, Stuttgart, 1986. [2] L. A. Kunyansky, Inverse Problems, 8:5 (1992), 809-819. [3] J. Radon, Ber. Verh. Konigl. Sachs. Ges. Wiss. Leipzig, 69 (1917), 262-277. [4] R. G. Novikov, Ark. Mat., 40:1 (2002), 145-167. [5] J. Boman, J. O. Strömberg, J. Geom. Anal., 14:2 (2004), 185-198. [6] S. Gindikin, Inverse Probl. Imaging, 4:4 (2010), 649-653. [7] L. T. Chang, IEEE Trans. Nucl. Sci., 25:1 (1978), 638-643. [8] K. Murase, H. Itoh, H. Mogami, M. Ishine, M. Kawamura, A. Lio, K. Hamamoto, Eur. J. Nucl. Med., 13 (1987), 55-62. [9] R. G. Novikov, arXiv: 1101.1725.

\section{P. Г. Новиков (R. G. Novikov)}

Международный институт теории прогноза землетрясений и математической геофизики РАН;

CNRS, Centre de Mathématiques Appliquées,

Ecole Polytechnique, France

E-mail: novikov@cmap.polytechnique.fr
Представлено С. П. Новиковым Принято редколлегией 01.02.2011 